\title{
Exposing drug industry funding of UK patient organisations
}

\section{(ㄷ) $\mathbb{( 1 )} \Theta$ OPEN ACCESS}

The "Who received funding?" section of this analysis by

Ozieranski and colleagues (BMJ 2019;365:11806, doi:10.1136/

bmj.11806) contained a typographical error. It should have read that "Most funding ( $£ 47466806,82.8 \%$ ) went to 314

multipurpose organisations ..." rather than 31 . This has been corrected on bmj.com. 